\title{
Hhip regulates tumor-stroma-mediated upregulation of tumor angiogenesis
}

\author{
Vijayendra Agrawal' ${ }^{2}$, Dong Young Kim and Young-Guen Kwon
}

Tumor growth is governed by the coordinated action of various types of cells that are present in the tumor environment. Fibroblasts, which constitute a major fraction of the stroma, participate actively in various signaling events and regulate tumor development and metastasis. The Hedgehog $(\mathrm{Hh})$ pathway plays an important role in promoting tumor malignancy via fibroblasts; however, the role of hedgehog interacting protein (hhip; inhibitor of Hh pathway) in tumor growth is poorly understood. Here we implanted B16F10 tumors in hhip+/- mice to study the tumor growth characteristics and the vascular phenotype. Furthermore, the mechanism involved in the observed phenomena was explored to reveal the role of hhip in tumor growth. The tumors that were implanted in hhip + / - mice exhibited accelerated growth and increased tumor angiogenesis. Although we observed a decrease in hypoxia, blood vessels still had abnormal phenotype. We found that increased Hh signaling in tumor fibroblasts induced a high expression of vascular endothelial growth factor (VEGF), which subsequently resulted in an increased proliferation of endothelial cells. Thus, the heterozygous knockdown of hhip in mice could affect Hh signaling in tumor fibroblasts, which could cause the increased production of the growth factor VEGF. This signaling, via a paracrine effect on endothelial cells, increased tumor vascular density.

Experimental \& Molecular Medicine (2017) 49, e289; doi:10.1038/emm.2016.139; published online 27 January 2017

\section{INTRODUCTION}

Tumors exhibit a co-operational and evolving coexistence of a number of cell types, including cancer cells, cancer initiating cells, endothelial cells, pericytes, immune cells, adipocytes and fibroblasts. These various cell types, of which fibroblasts constitute a major fraction, are embedded in the extracellular matrix, the composition of which is determined by a multitude of factors (such as growth factors, chemokines and cytokines) that are released by the different cell types. These autocrine/ paracrine acting factors have a determinant role in the survival and progression of tumors. ${ }^{1-3}$

Various growth factors are released by the tumor cells in their niche and act on several other cell types to promote tumor growth. One of the factors, sonic hedgehog (shh), has been widely implicated in a variety of cancers. ${ }^{4,5}$ shh, when it is released from tumor cells, binds to its cognate receptor patched (ptch1) in stroma, thereby causing the release of smoothened (Smo), which subsequently leads to the activation of transcription factor Gli. Gli activation results in the increased production of various growth factors, such as vascular endothelial growth factor (VEGF), hepatocyte growth factor (HGF), platelet-derived growth factor (PDGF) and angiopoietins, ${ }^{6}$ which contribute to tumor cellular proliferation, metastasis and resistance to therapy. ${ }^{7}$ Activation of this pathway is limited by hedgehog binding proteins, such as hedgehog interacting protein (hhip). ${ }^{8}$ Activating mutations in $\mathrm{Hh}$ pathway genes are associated with cancers in several tissues, such as brain, lung, mammary gland, prostate and skin. ${ }^{9-12}$ In addition, the Hh pathway promotes survival, proliferation and the epithelialto-mesenchymal (EMT) transition of tumor cells, causing metastasis. $^{13-15}$

The Hh pathway inhibitor hhip is highly expressed in endothelial cells; however, its expression is sharply reduced in epithelial cells and fibroblasts, during angiogenesis and in tumor tissues. ${ }^{16}$ Deletion of hhip from stromal cells promotes the proliferation of leukemic cells. ${ }^{17}$ Loss-offunction hhip mutations mainly affect lung and endochondral skeleton in mouse, with postnatal lethality in homozygous knockout mice as a result of respiratory failure. ${ }^{18}$ These studies show that hhip is a highly specific and potent endogenous inhibitor of Hh pathway and this pathway has a crucial role in controlling tumor growth and angiogenesis,

Department of Biochemistry, College of Life Science and Biotechnology, Yonsei University, Seoul, Republic of Korea

2Current address: Sanford-Burnham Medical Research Institute at Lake Nona, 6400 Sanger Road, Orlando, FL 32827, USA.

Correspondence: Professor Dr Y-G Kwon, Department of Biochemistry, College of Life Science and Biotechnology, Yonsei University, Seoul 120-749, Republic of Korea.

E-mail: ygkwon@yonsei.ac.kr

Received 26 May 2016; revised 21 August 2016; accepted 22 August 2016 
yet the role of hhip in tumor angiogenesis is not well understood.

Here we used hhip heterozygous mice to investigate the potential effect of hhip on tumor blood vessels. Reduced hhip expression in mice resulted in increased Hh activity in tumor fibroblasts. This effect resulted in an increased secretion of VEGF from fibroblasts, which exhibited a paracrine effect on endothelial cell proliferation and increased the vascular density in tumors. Although reduced hypoxia was observed in the tumor environment, the vessels resulting from the downregulation of hhip still had abnormal phenotype.

\section{MATERIALS AND METHODS}

\section{Mice}

C57BL6 male mice (5-7 weeks old) were purchased from Daehan Biolink (Seoul, Korea), and Hhip knockout mice were purchased from The Jackson Laboratory (Davis, CA, USA). Genotyping of hhip mice was performed using the following primers: 5'-ATCCTCCC GCGTCCTGCCCTTG- $3^{\prime}$ and 5'-TCAGGCACCGTCTCCTTCTCGC $-3^{\prime}$ for the wild-type gene; $5^{\prime}$-ATCCTCCCGCGTCCTGCCCTTG-3' and $5^{\prime}$-TCGCGTCTGGCCTTCCTGTAGC-3' for the mutant gene. The wild-type gene produced a PCR product of $169 \mathrm{bp}$, whereas the mutant gene produced a 460-bp product.

All mice were maintained in a laminar airflow cabinet under specific pathogen-free conditions. The facilities were approved by the Association of Assessment and Accreditation of Laboratory Animal Care, and animal experiments were conducted under the institutional guidelines established for the Animal Core Facility at Yonsei University College of Medicine, with approval from the institutional care and use committee.

\section{LacZ staining}

Mouse embryos were dissected from extraembryonic membranes and fixed in fixative solution ( 5 mm EGTA, $0.2 \%$ glutaraldehyde and $2 \mathrm{~mm}$ $\mathrm{MgCl}_{2}$ in $0.1 \mathrm{M}$ phosphate buffer, $\mathrm{pH} 7.3$ ) for 30 minutes. Embryos were rinsed in detergent rinse $\left(2 \mathrm{mM} \mathrm{MgCl}_{2}, 0.02 \% \mathrm{NP}-40\right.$ and $0.01 \%$ sodium deoxycholate in $0.1 \mathrm{~m}$ phosphate buffer, $\mathrm{pH}$ 7.3) for $15 \mathrm{~min}$ thrice at room temperature and were then incubated in embryo staining solution $\left(2 \mathrm{~mm} \mathrm{MgCl}_{2}, 0.02 \% \mathrm{NP}-40,5 \mathrm{~mm}\right.$ potassium ferricyanide, $5 \mathrm{~mm}$ potassium ferrocyanide, $0.01 \%$ sodium deoxycholate, $1 \mathrm{mg} \mathrm{ml}^{-1} \mathrm{X}$-gal in $0.1 \mathrm{~m}$ phosphate buffer, $\mathrm{pH} 7.3$ ) at $37^{\circ} \mathrm{C}$ for 4 to $5 \mathrm{~h}$. Stained embryos were stored in $70 \%$ ethanol at $4{ }^{\circ} \mathrm{C}$ and later photographed.

\section{Tumor model}

B16F10 cells $\left(5 \times 10^{5}\right.$ cells per $\left.100 \mu \mathrm{l}\right)$ were subcutaneously implanted on the lateral flank of 8-week-old hhip+/ - and littermate wild-type mice. Tumor volume was measured with calipers and calculated as width ${ }^{2} \times$ length $\times 0.523$.

\section{Tumor permeability and vascular perfusion}

Tumor permeability was assessed by the Evans blue and the fluorescein isothiocyanate (FITC)-dextran assays as previously described. ${ }^{19}$ Evans blue $\left(50 \mathrm{mg} \mathrm{kg}^{-1}\right)$ was injected intravenously, and tumors were excised $30 \mathrm{~min}$ later. Tumors were dried at $60^{\circ} \mathrm{C}$ for $16 \mathrm{~h}$, and then dye was extracted with $1 \mathrm{ml}$ formamide at $55^{\circ} \mathrm{C}$ for $16 \mathrm{~h}$. Absorbance was measured at $620 \mathrm{~nm}$.

Vascular leakage visualization with FITC-dextran was achieved by an intravenous injection of $3 \mathrm{mg}$ per mouse FITC-dextran $(40-\mathrm{kDa}$;
Sigma Aldrich, St Louis, MO, USA) $10 \mathrm{~min}$ before tumor capture. Tumors were then fixed briefly in $4 \%$ paraformaldehyde, and cryosections were made to observe vascular leakage by fluorescence microscopy.

\section{Histology and immunostaining}

To evaluate tumor histology, tumors were excised after perfusing the mice with $1 \%$ paraformaldehyde and were briefly incubated in 4\% paraformaldehyde. After an overnight incubation in 15 and $30 \%$ sucrose each, the specimens were embedded in optimal cutting temperature compound and sectioned at $30 \mu \mathrm{m}$ thickness. Staining was performed as previously described ${ }^{20}$ by incubation with one of the following antibodies: rat anti-CD31 (1:100; BD Pharmingen, Seoul, Korea), goat anti-VE-cadherin (1:100; Santa Cruz, Santa Cruz, CA, USA), mouse anti-Ki67 (1:100; BD Pharmingen), rabbit anti-NG2 (1:500; Millipore, Seoul, Korea), rabbit anti-FAP (1:100; Novus Biologicals, Littleton, CO, USA), mouse anti-hhip (1:100; Novus Biologicals), rabbit anti-Ptch1 (1:100; Santa Cruz) or rabbit antiVEGF (1:200; Abcam, Cambridge, UK). The sections were then incubated in Alexa Fluor-conjugated secondary antibodies. Nuclear staining was performed with 4',6-diamidino-2-phenylindole (DAPI, $\left.1 \mathrm{ng} \mathrm{ml}^{-1}\right)$. Sections were then photographed with a confocal microscope (Zeiss LSM 510, Oberkochen, Germany).

Hypoxia was detected by the pimonidazole adduct formation caused by an intravenous injection of $75 \mathrm{mg} \mathrm{kg}^{-1}$ pimonidazole (Hypoxyprobe-1, Chemicon, Binerica, MA, USA) $1 \mathrm{~h}$ before tumor capture. Adducts were stained with a monoclonal antibody directed against pimonidazole (Hypoxyprobe-1 kit, Chemicon).

\section{Western blotting}

Western blot analysis was performed as previously described. ${ }^{21}$ Tumor specimens $\left(1 \mathrm{~mm}^{3}\right.$ tissue) were homogenized in RIPA buffer and centrifuged. The proteins obtained from the supernatants were electrophoresed on $8 \%$ sodium dodecyl sulfate-polyacrylamide gels and transferred to nitrocellulose membranes. The primary antibody used to visualize the protein bands was rabbit anti-Ptch1 (Santa Cruz). Horseradish peroxidase-conjugated secondary antibody was obtained from ThermoScientific (Rockford, IL, USA). Protein bands were detected using an ECL Western blotting detection kit (Amersham Biosciences, San Francisco, CA, USA) according to the manufacturer's instructions.

\section{Real-time PCR}

Total RNA was isolated from B16F10 tumor samples using RNeasy Plus Universal kit (Qiagen, Valencia, CA, USA) according to the manufacturer's instructions. cDNA was synthesized, and real-time PCR was performed on a pikoreal 96 machine (ThermoScientific) using Maxima SYBR green qPCR master mix (ThermoScientific). Gene-specific primers were used at a final concentration of $0.5 \mu \mathrm{M}$, and their sequences are listed in Supplementary Table 1. All qPCR assays were performed in triplicate in at least three independent experiments using two different samples.

\section{Statistical analysis}

All statistical analyses were performed using GraphPad Prism (version 5.0; GraphPad Software, La Jolla, CA, USA). Tests for statistical significance were two-sided, and probability values $<0.05$ were considered significant. Student's $t$-test or analysis of variance was used to compare mean values, and the results are presented as the mean \pm s.e.m. or s.d. Image analysis was performed using the ImageJ program. 
a

Hhip embryo (E11.5) LacZ staining
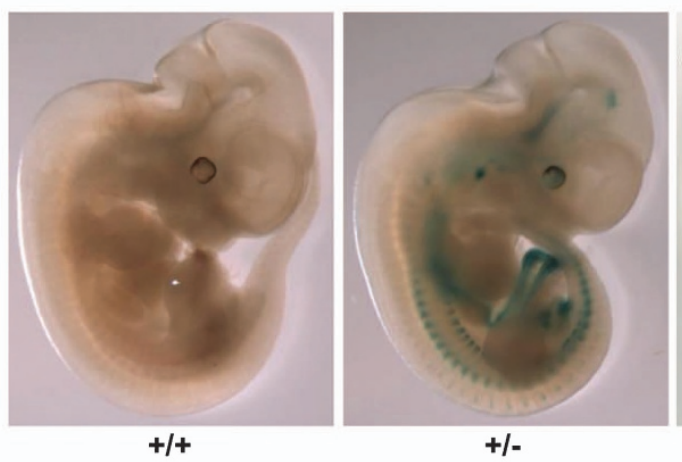

$+1-$

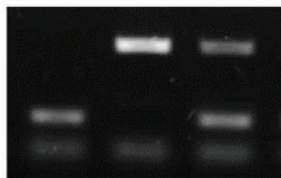

Mutant band

Wild band

Hhip embryo genotyping

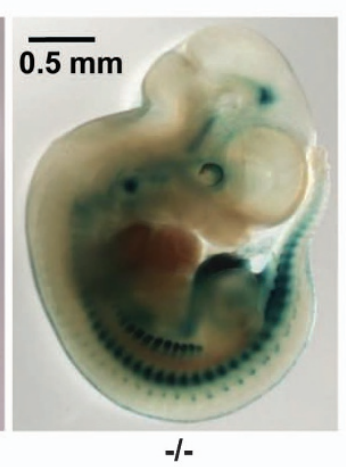

b
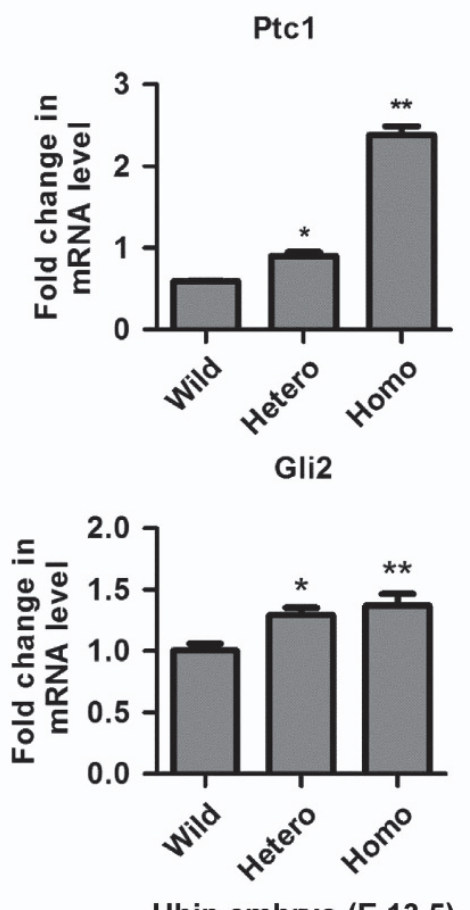

C

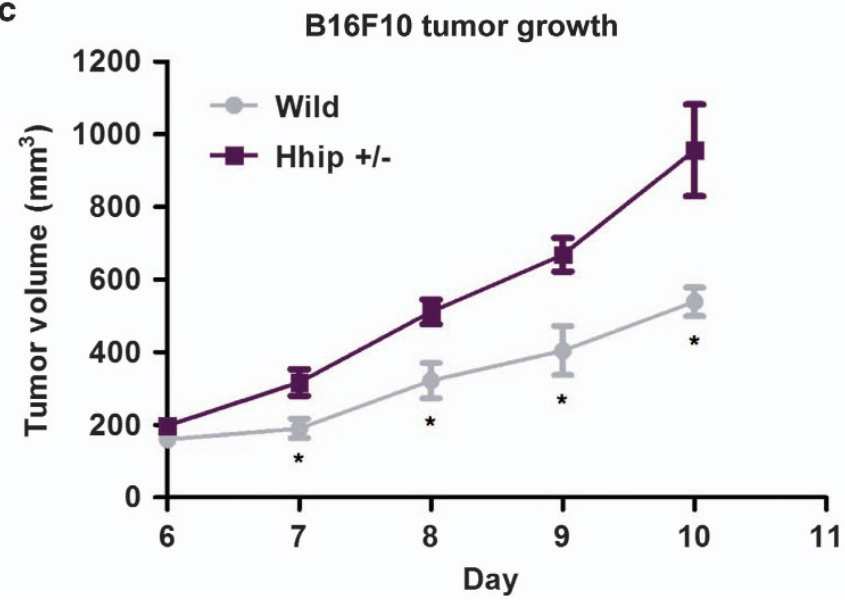

d

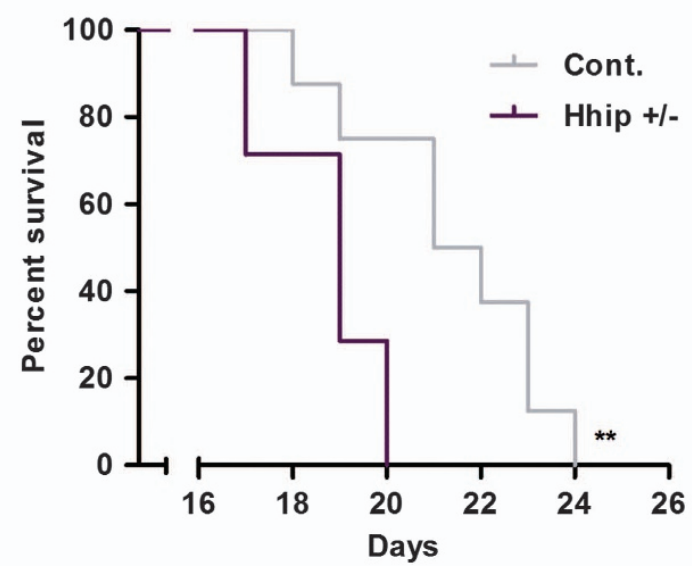

Figure 1 (a) Confirmation of hetero/homozygosity of hhip knockout embryos by LacZ staining and RT-PCR. (b) Activation of Hh pathway target gene Ptch1 and the downstream signaling molecule Gli2 in knockout embryos. (c) Acceleration of B16F10 tumor growth in hhip+/mice correlated with (d) reduced survival of hhipt/ - mice.

\section{Ethics statement}

This investigation was conducted in accordance with the ethical standards and according to the Declaration of Helsinki and national and international guidelines. The study was approved by the Animal Core Facility at Yonsei University College of Medicine.

\section{RESULTS}

Tumor growth was enhanced in Hhip + / - mice

Given that homozygous knockout of hhip resulted in postnatal lethality in mice, we used hhip heterozygous mice for the experiments. Heterozygosity was confirmed at the embryonic stage by LacZ staining and genotyping; in adult mice, heterozygosity was diagnosed by genotyping (Figure 1a). qRT-PCR of RNA isolated from whole embryos revealed that the expression of Ptch1 and Gli2, targets as well of components of the hhip pathway, was increased in homo/hetero knockout embryos (Figure 1b). In adult mice, increased Ptch1 expression was observed in lungs, which are the target organs of the hhip pathway (Supplementary Figure 1a).

First, we analyzed the blood vessel phenotypes in hhip mutant embryos and their corresponding yolk sacs. However, no obvious difference was observed (Supplementary Figure 1b). This finding prompted us to redirect our focus on pathological angiogenesis in adult mice. B16F10 tumor cells were injected 
subcutaneously in the flank of 8-week-old hhip + - - mice and their wild-type littermates. Rapid tumor growth was noted in hhip + / - mice compared with wild type (Figure 1c). This enhancement in tumor growth was also reflected in the reduced survival of tumor-bearing hhip + / - mice (Figure 1d).

Increased tumor growth might result from tumor cell proliferation as well as proliferation of tumor blood vessels. Thus, we immunostained size-matched tumor sections from wild-type and hhip+/ - mice for the proliferation marker Ki67. Compared with wild-type tumors, most of the vessels in hhip + / - mice tumors exhibited strong positivity for Ki67 (Figures 2a and b). Although the extent of proliferation in tumor cells did not differ between the groups, an enhancement in vascular proliferation was demonstrated by significantly increased vascular density in hhip + / - mice tumors (Figures $2 \mathrm{c}$ and d). This increase in vascular proliferation was also demonstrated by ex vivo aortic ring sprout assays (Supplementary Figure 2). Hence, a reduction of hhip expression in mice could positively affect pathological angiogenesis in tumors.

\section{Tumor vessels in hhip $+/$ - mice tumors were abnormal in nature}

We next investigated the phenotype of blood vessels in tumors of hhip + / - mice. Immunostaining for the hypoxic region in tumors showed that hypoxia was reduced in heterozygous mice (Figures $2 \mathrm{c}$ and $\mathrm{e}$ ). This soothing effect upon the tumor environment might be attributed to increased vascular density, which could enhance the oxygen supply to the tumor tissue. A reduction in hypoxia might also indicate that the tumor blood vessels in hhip + / - mice have reduced vascular permeability. However, analysis of vascular leakage by Evans blue and the FITC-dextran leakage method showed that blood vessels from hhip +/ - tumors had significantly increased vascular leakage (Figure $2 \mathrm{f}-\mathrm{h}$ ). Importantly, immunostaining of hhip+l- tumor sections for the junctional protein VE-cadherin exhibited punctuated, non-continuous lining of VE-cadherin along the vessel wall, similar to that of wild-type tumors (Supplementary Figure 3). This finding suggests that the observed increase in leakiness was a result of a combined effect of an increase in vessel number and individual vessel leakiness rather than an increase in the leakiness of individual vessels.

We also analyzed the ratio of perfused blood vessels in tumor sections from wild and hhip $+/-$ mice. In accordance with the increase in vascular leakiness, tumors from hhip + / mice exhibited reduced vascular patency (Figures $2 \mathrm{i}$ and $\mathrm{j}$ ). Analysis of the pericyte coverage of blood vessels revealed that the extent of blood vessel coverage by pericytes was also reduced in tumors from hhip $+/-$ mice (Figures $2 \mathrm{k}$ and 1 ). These data suggest that although hhip + / - mice tumors had an increased number of blood vessels, the individual vessels still had abnormal phenotype.

\section{Hedgehog signaling was upregulated in tumor fibroblasts from hhip + / - mice}

Because we observed significant differences in the tumor blood vessels of hhip + / - mice compared with wild type, we sought to identify the mechanism behind this finding. First, we analyzed the tumor tissues from wild mice to identify the cell types that exhibited hhip expression. Immunostaining of tumor slides from wild-type mice for CD31 and hhip showed that the expression of hhip was primarily located in extra-tumoral tissue. Inside the tumor, blood vessels and other cells exhibited hhip expression, albeit at lower levels (Supplementary Figure 4a). To identify the nature of extravascular cells expressing hhip, we co-stained the tumor sections with fibroblast marker (S100A4) and hhip. These data revealed that the cells expressing hhip were in fact fibroblasts (Figure 3a). Co-staining of wild-type tumor sections with Ptch1 (one of the targets of Hh signaling) and hhip also demonstrated that cells with Ptch1 expression were the same as those that expressed hhip, that is, fibroblasts (Figure 3b). Next, we analyzed the Ptch1 expression levels in tumor sections from wild and hhip $+/-$ mice. High-magnification images clearly showed that the fibroblasts around blood vessels had increased Ptch1 expression in hhip + / - tumors (Figures $3 \mathrm{c}$ and d). Hence, we hypothesize that the increased hedgehog activity in fibroblasts surrounding blood vessels might explain the observed vascular difference in hhip+/ - mice tumors.

\section{Fibroblasts from Hhip $+/-$ mice tumors exhibit increased VEGF secretion}

Because we observed increased Hh signaling in tumor fibroblasts of hhip + / - mice, we sought to identify the downstream target molecule. Previous studies have shown that fibroblasts exhibit canonical Hh signaling in which angiopoietins and VEGF are secreted in response to increased Hh signaling. ${ }^{6}$ Immunofluorescence analysis of tumor sections revealed that VEGF expression was significantly increased in hhip+/tumors and that the expression was confined around fibroblast cells (Figures 4a and b). qRT-PCR analysis of samples from wild-type and hhip + - - tumors confirmed the staining results (Figure 4c). We also assessed the expression of other downstream targets of canonical Hh signaling, such as angiopoietins in the RNA samples isolated from whole tumors of wild and hhip+/ - mice. However, no significant difference in gene expression was observed (Supplementary Figure $4 \mathrm{~b}$ ). Thus, our data suggest that increased angiogenesis observed in the B16F10 tumor grown in hhip+/ - mice might be due to the increased secretion of VEGF from tumor fibroblasts (Figure 5).

\section{DISCUSSION}

Here we demonstrated that the heterozygous deletion of hhip from host mice could affect hedgehog signaling in tumorassociated-fibroblasts (TAFs), leading to increased pathological angiogenesis. The absence of a single copy of hhip in the host genome mostly affected stromal cells in the tumor niche. Enhanced unchecked activity of the Hh pathway led to the increased secretion of the growth factor VEGF from fibroblasts, which affected the nearby endothelial cells and led to elevated vascular density.

TAFs are distinctive and the major component of the tumor microenvironment, a niche that has a significant role in tumor 

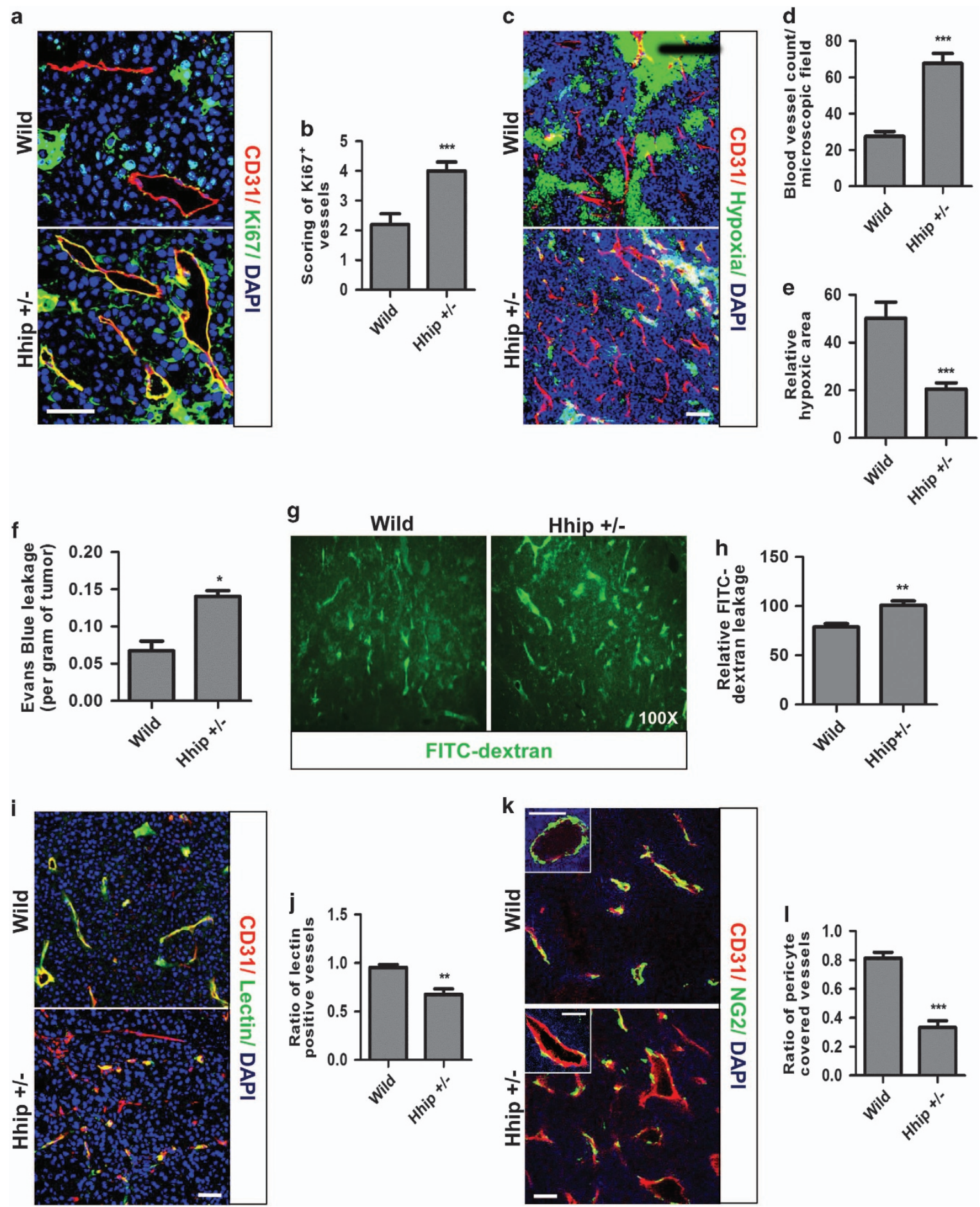

Figure 2 (a, b) Immunostaining of tumor sections to identify proliferating cells. (c, e) Tumor sections were stained for pimonidazole to identify hypoxia-rich regions. (d) Blood vessel count was obtained from sections stained in c. (f) Quantitation of tumor vascular leakage by the Evans blue method. ( $\mathbf{g}, \mathbf{h})$ Vascular leakage was visualized by FITC-dextran angiography and quantified. (i, j) Tumor sections were co-stained with CD31 and lectin to investigate vascular patency. (k, I) Pericyte coverage of blood vessels was assessed using NG2 staining. Representative figures from five different tumors are presented. Scale bar, $50 \mu \mathrm{m}$.

growth and pathological angiogenesis. TAFs play an important role in tuning the various signaling events that occur in the tumor environment. ${ }^{22,23}$ TAFs have a typical genetic signature compared with fibroblasts from normal tissues. ${ }^{2,3,24}$ Previous studies with breast carcinoma have shown that these TAFs could promote the growth of tumor cells and recruit EPCs to tumors in part through secretion of stromal cell-derived factor (SDF)-1..$^{25}$ TAFs also promote tumor progression through 
a

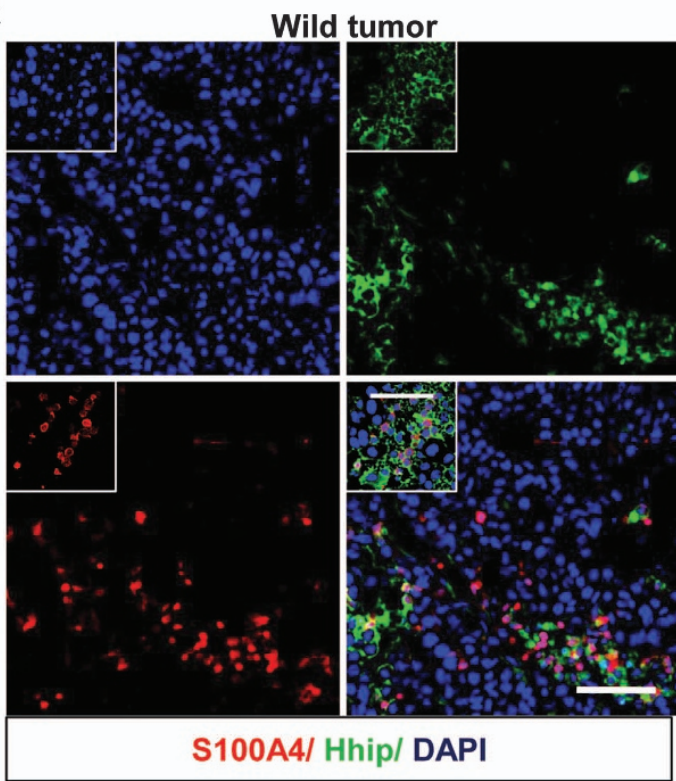

b

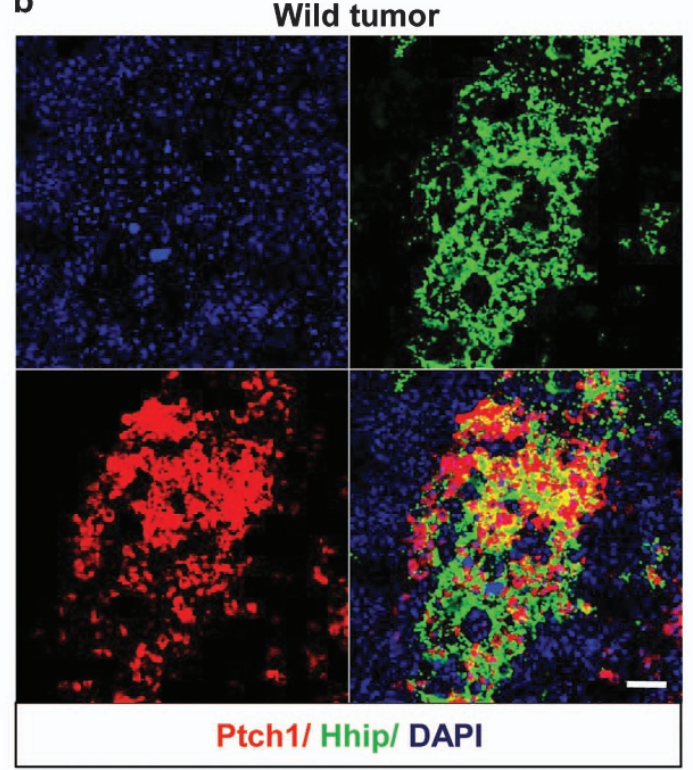

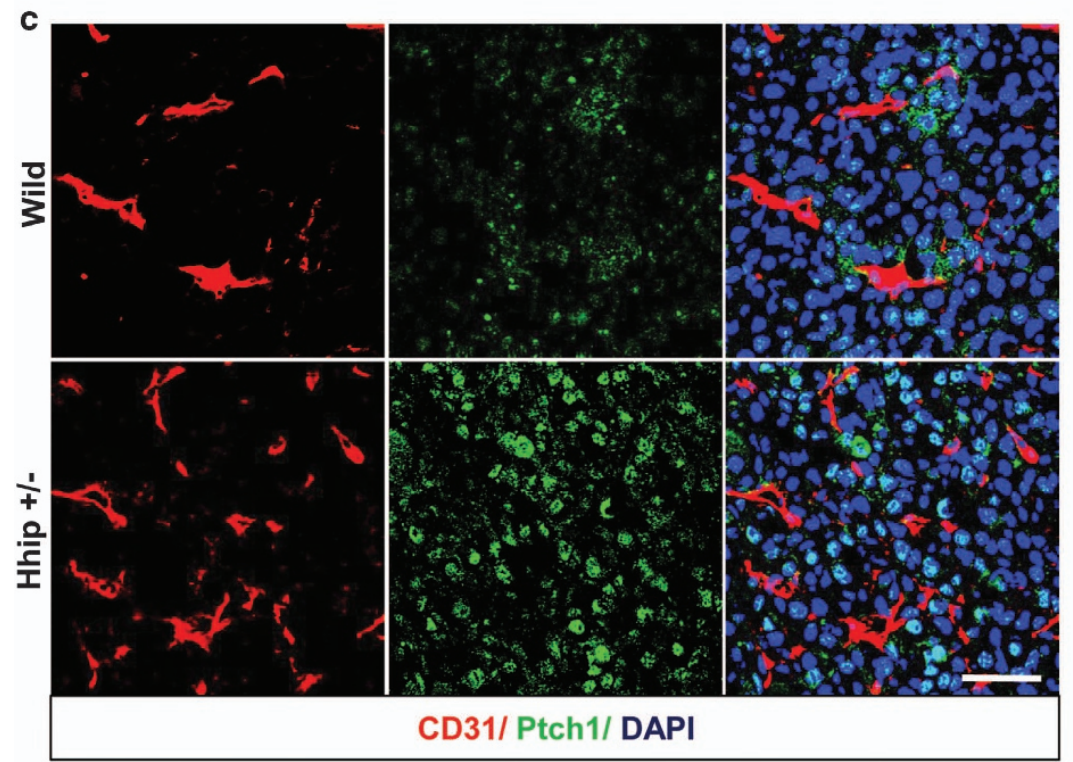

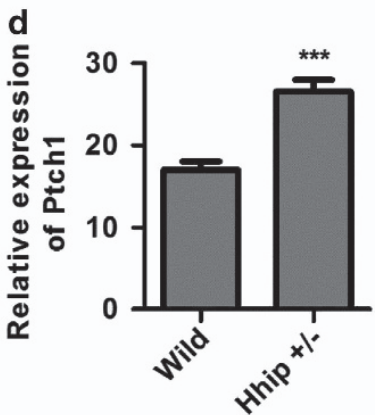

Figure 3 (a) Wild-type tumor sections stained with fibroblast marker (FAP) and hhip (b) or Ptch1 and hhip to identify the cells that express hhip and the response to Hh signaling. (c, d) Tumor sections from hhip+/- and wild-type mice were immunostained with Ptch1 to examine elevated Hh signaling. Representative figures from five different tumors are presented. Scale bar, $50 \mu \mathrm{m}$.

secretion of multiple factors and MMPs, inducing stemness, EMT and epigenetic changes. ${ }^{26,27}$ In our study, we found that TAFs were the major cell population affected by deletion of hhip in host mice. Hh signaling, as indicated by the elevated expression of Ptch1, was specifically upregulated in TAFs, which led to observed difference in blood vessel growth. Our observation is also consistent with a previous report in which ectopic expression of shh was found to enhance tumor angiogenesis via canonical signaling in stromal cells. ${ }^{6}$

Hhip is the endogenous inhibitor of hedgehog pathway, a pathway that impacts various stages of tumor growth. The Hh pathway is an active participant in cancer development, progression and metastasis. ${ }^{4}$ Gli-Hh signaling has also been reported to enhance the proliferation of melanoma cells. ${ }^{28}$ Glioma cell proliferation is supported by one of the $\mathrm{Hh}$ pathway targets, cyclin D2;, 59,30 similarly, breast cancer metastasis is promoted by one of the targets of the Hh pathway, CYR61. ${ }^{31}$ The Hh pathway promotes the expression of VEGF and angiopoietins in tumor environment, thereby promoting the proliferation of blood vessels and promoting tumor malignancy. ${ }^{6}$ The Hh pathway also supports tumor cell invasion by increasing cell motility and MMP expression and by directly targeting genes associated with EMT, such as Snai1. ${ }^{13,32}$ Furthermore, the pathway supports cancer genomic instability and inflammation. ${ }^{33}$ 
a
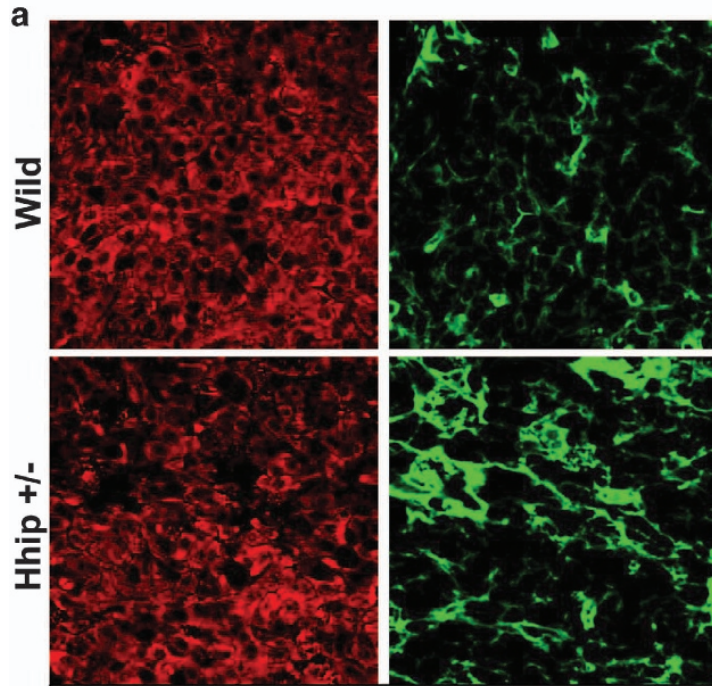
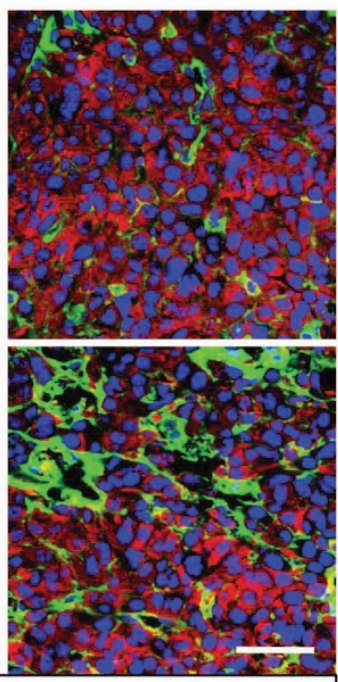

FAPI VEGF/ DAPI
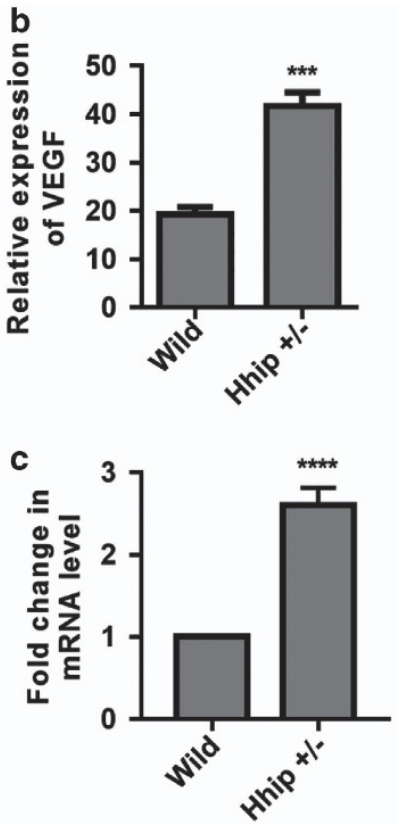

Figure 4 (a, b) Immunostaining of tumor sections to confirm elevated expression of VEGF around fibroblasts in hhip $+/-$ mice. Representative figures from five different tumors are presented. Scale bar, $50 \mu \mathrm{m}$. (c) qRT-PCR of tumor samples from wild-type and hhip+l- mice $(n=5)$.

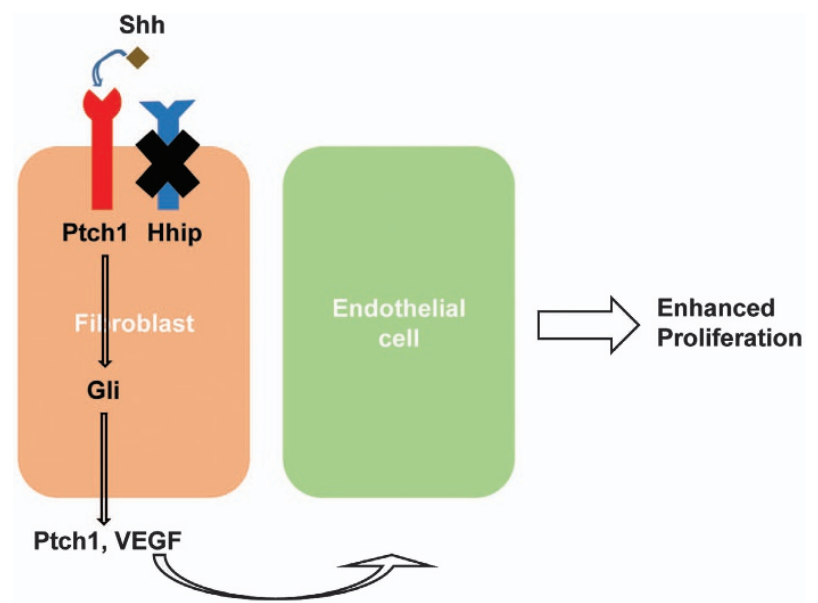

Figure 5 Graphical presentation depicting hhip deletion-mediated increase in endothelial cell proliferation in tumor tissues.

As a negative regulator of the Hh pathway, Hhip is downregulated as a result of promoter hypermethylation in a number of cancers. ${ }^{34-36}$ Knockdown of hhip in bone marrow-derived stromal cells supports the proliferation of Smo+ leukemic cells. ${ }^{17}$ However, the direct effect of hhip knockdown on endothelial cells is not clear. Here we heterozygously knocked out hhip in mice and studied its effect on both physiologic and pathologic angiogenesis. The absence of a single copy of functional hhip had a minimal effect on vascular growth in embryos and yolk sacs, as demonstrated by our observation; however, the effect on tumor angiogenesis was significant. Although no improvement in vascular normalization was observed, the vascular density increased in hhip+/ - mice tumors. Hhip deletion could impact tumor metastasis; however, further investigations are needed.

Factors affecting the tumor microenvironment could have great impact on tumor growth and metastasis. Our study is the first to demonstrate that the systemic deletion of hhip could have a positive effect on tumor angiogenesis. This investigation furthers our understanding of the mechanism underlying the impact of the Hh pathway on tumor growth and supports the idea that tumor fibroblasts are promising targets for tumor therapy.

\section{CONFLICT OF INTEREST}

The authors declare no conflict of interest.

\section{ACKNOWLEDGEMENTS}

This research was supported by Basic Science Research Program through the National Research Foundation of Korea (NRF) grant funded by the Korea government (MSIP) (NRF2015R1A2A1A05001859) and (NRF-2013M3A9B6046563), and by the Bio and Medical Technology Development Program of the National Research Foundation of Korea (NRF) funded by the Korea government (MSIP) (NRF-2015M3A9B6066967).

1 Alphonso A, Alahari SK. Stromal cells and integrins: conforming to the needs of the tumor microenvironment. Neoplasia 2009; 11: 1264-1271.

2 Mao Y, Keller ET, Garfield DH, Shen K, Wang J. Stromal cells in tumor microenvironment and breast cancer. Cancer Metastasis Rev 2013; 32: 303-315.

3 Cirri P, Chiarugi P. Cancer-associated-fibroblasts and tumour cells: a diabolic liaison driving cancer progression. Cancer Metastasis Rev 2012; 31: 195-208. 
4 Harris LG, Samant RS, Shevde LA. Hedgehog signaling: networking to nurture a promalignant tumor microenvironment. Mol Cancer Res 2011; 9: 1165-1174.

5 Shahi MH, Rey JA, Castresana JS. The sonic hedgehog-GLII signaling pathway in brain tumor development. Expert Opin Ther Targets 2012; 16: 1227-1238.

6 Chen W, Tang T, Eastham-Anderson J, Dunlap D, Alicke B, Nannini M et al. Canonical hedgehog signaling augments tumor angiogenesis by induction of VEGF-A in stromal perivascular cells. Proc Natl Acad Sci USA 2011; 108 . 9589-9594.

7 Li X, Ma Q, Duan W, Liu H, Xu H, Wu E. Paracrine sonic hedgehog signaling derived from tumor epithelial cells: a key regulator in the pancreatic tumor microenvironment. Crit Rev Eukaryot Gene Expr 2012; 22: 97-108.

8 Chuang PT, McMahon AP. Vertebrate Hedgehog signalling modulated by induction of a Hedgehog-binding protein. Nature 1999; 397: 617-621.

9 Sekulic A, Mangold AR, Northfelt DW, LoRusso PM. Advanced basal cell carcinoma of the skin: targeting the hedgehog pathway. Curr Opin Oncol 2013; 25: 218-223.

10 Gupta S, Takebe N, Lorusso P. Targeting the Hedgehog pathway in cancer. Ther Adv Med Oncol 2010; 2: 237-250.

11 Wang XD, Inzunza H, Chang H, Qi Z, Hu B, Malone D et al. Mutations in the hedgehog pathway genes $\mathrm{SMO}$ and $\mathrm{PTCH} 1$ in human gastric tumors. PLOS ONE 2013; 8: e54415.

12 Hui M, Cazet A, Nair R, Watkins DN, O'Toole SA, Swarbrick A. The Hedgehog signalling pathway in breast development, carcinogenesis and cancer therapy. Breast Cancer Res 2013; 15: 203.

13 Ke Z, Caiping S, Qing Z, Xiaojing W. Sonic hedgehog-Gli1 signals promote epithelial-mesenchymal transition in ovarian cancer by mediating PI3K/AKT pathway. Med Oncol 2015; 32: 368.

14 Steinway SN, Zanudo JG, Ding W, Rountree CB, Feith DJ, Loughran TP Jr et al. Network modeling of TGFbeta signaling in hepatocellular carcinoma epithelial-to-mesenchymal transition reveals joint sonic hedgehog and Wnt pathway activation. Cancer Res 2014; 74: 5963-5977.

15 Islam SS, Mokhtari RB, Noman AS, Uddin M, Rahman MZ, Azadi MA et al. Sonic hedgehog (Shh) signaling promotes tumorigenicity and stemness via activation of epithelial-to-mesenchymal transition (EMT) in bladder cancer. Mol Carcinog 2015; 55: 537-551.

16 Olsen CL, Hsu PP, Glienke J, Rubanyi GM, Brooks AR. Hedgehoginteracting protein is highly expressed in endothelial cells but downregulated during angiogenesis and in several human tumors. BMC Cancer 2004; 4: 43.

17 Kobune M, lyama S, Kikuchi S, Horiguchi H, Sato T, Murase K et al. Stromal cells expressing hedgehog-interacting protein regulate the proliferation of myeloid neoplasms. Blood Cancer J 2012; 2: e87.

18 Chuang PT, Kawcak T, McMahon AP. Feedback control of mammalian Hedgehog signaling by the Hedgehog-binding protein, Hip1, modulates Fgf signaling during branching morphogenesis of the lung. Genes Dev 2003; 17: 342-347.

19 Gratton JP, Lin MI, Yu J, Weiss ED, Jiang ZL, Fairchild TA et al. Selective inhibition of tumor microvascular permeability by cavtratin blocks tumor progression in mice. Cancer Cell 2003; 4: 31-39.

20 Winkler F, Kozin SV, Tong RT, Chae SS, Booth MF, Garkavtsev I et al. Kinetics of vascular normalization by VEGFR2 blockade governs brain tumor response to radiation: role of oxygenation, angiopoietin-1, and matrix metalloproteinases. Cancer Cell 2004; 6: 553-563.

21 Maharjan S, Lee S, Agrawal V, Choi HJ, Maeng YS, Kim K et al. Sac-0601 prevents retinal vascular leakage in a mouse model of diabetic retinopathy. Eur J Pharmacol 2011; 657: 35-40.

22 Augsten M. Cancer-associated fibroblasts as another polarized cell type of the tumor microenvironment. Front Oncol 2014; 4: 62.
23 Han Y, Zhang Y, Jia T, Sun Y. Molecular mechanism underlying the tumorpromoting functions of carcinoma-associated fibroblasts. Tumour Biol 2015; 36: 1385-1394.

24 Ohlund D, Elyada E, Tuveson D. Fibroblast heterogeneity in the cancer wound. J Exp Med 2014; 211: 1503-1523.

25 Orimo A, Gupta PB, Sgroi DC, Arenzana-Seisdedos F, Delaunay T, Naeem R et al. Stromal fibroblasts present in invasive human breast carcinomas promote tumor growth and angiogenesis through elevated SDF-1/CXCL12 secretion. Cell 2005; 121: 335-348.

26 Damhofer H, Medema JP, Veenstra VL, Badea L, Popescu I, Roelink H et al. Assessment of the stromal contribution to Sonic Hedgehog-dependent pancreatic adenocarcinoma. Mol Oncol 2013; 7: 1031-1042.

27 Kharaishvili G, Simkova D, Bouchalova K, Gachechiladze M, Narsia N, Bouchal J. The role of cancer-associated fibroblasts, solid stress and other microenvironmental factors in tumor progression and therapy resistance. Cancer Cell Int 2014; 14: 41.

28 Geng L, Cuneo KC, Cooper MK, Wang H, Sekhar K, Fu A et al. Hedgehog signaling in the murine melanoma microenvironment. Angiogenesis 2007; 10: 259-267.

29 Koyama-Nasu R, Nasu-Nishimura Y, Todo T, Ino Y, Saito N, Aburatani H et al. The critical role of cyclin D2 in cell cycle progression and tumorigenicity of glioblastoma stem cells. Oncogene 2013; 32: 3840-3845.

30 Shahi MH, Afzal M, Sinha S, Eberhart CG, Rey JA, Fan X et al. Regulation of sonic hedgehog-GLI1 downstream target genes PTCH1, Cyclin D2, Plakoglobin, PAX6 and NKX2.2 and their epigenetic status in medulloblastoma and astrocytoma. BMC Cancer 2010; 10: 614.

31 Harris LG, Pannell LK, Singh S, Samant RS, Shevde LA. Increased vascularity and spontaneous metastasis of breast cancer by hedgehog signaling mediated upregulation of cyr61. Oncogene 2012; 31: 3370-3380.

32 Choe C, Shin YS, Kim SH, Jeon MJ, Choi SJ, Lee J et al. Tumor-stromal interactions with direct cell contacts enhance motility of non-small cell lung cancer cells through the hedgehog signaling pathway. Anticancer Res 2013; 33: 3715-3723.

33 Lauth M, Toftgard R. Think inside the BOCs: a mechanism underlying medulloblastoma progression. Dev Cell 2014; 31: 1-2.

34 Tada M, Kanai F, Tanaka Y, Tateishi K, Ohta M, Asaoka Y et al. Downregulation of hedgehog-interacting protein through genetic and epigenetic alterations in human hepatocellular carcinoma. Clin Cancer Res 2008; 14: 3768-3776.

35 Taniguchi H, Yamamoto H, Akutsu N, Nosho K, Adachi Y, Imai K et al. Transcriptional silencing of hedgehog-interacting protein by $\mathrm{CpG}$ hypermethylation and chromatic structure in human gastrointestinal cancer. J Pathol 2007; 213: 131-139.

36 Martin ST, Sato N, Dhara S, Chang R, Hustinx SR, Abe T et al. Aberrant methylation of the Human Hedgehog interacting protein (HHIP) gene in pancreatic neoplasms. Cancer Biol Ther 2005; 4: 728-733.

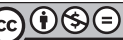

This work is licensed under a Creative Commons Attribution-NonCommercial-NoDerivs 4.0 International License. The images or other third party material in this article are included in the article's Creative Commons license, unless indicated otherwise in the credit line; if the material is not included under the Creative Commons license, users will need to obtain permission from the license holder to reproduce the material. To view a copy of this license, visit http://creativecommons.org/licenses/by-nc-nd/4.0/

Supplementary Information accompanies the paper on Experimental \& Molecular Medicine website (http://www.nature.com/emm) 